\title{
Diagnóstico serológico de Anaplasma phagocytophilum en caballos Fina Sangre de Carrera pertenecientes al Valparaíso Sporting Club Viña del Mar
}

\author{
Patricia Rodríguez Jorquera ${ }^{1}$, Cristian Conejeros Ortiz $^{2}$ \\ ${ }^{1}$ Facultad de Ciencias Naturales Universidad Santo Tomas. \\ ${ }^{2}$ Escuela de Ciencias Veterinarias Universidad Viña del Mar. Email: cristianconejeros.o@gmail.com.
}

\begin{abstract}
Resumen
El creciente desarrollo de las disciplinas deportivas equinas, ha obligado a procurar un estado sanitario óptimo para su participación. Es por eso que evaluar e identificar las potenciales patologías que pudiesen alterar el desempeño de los ejemplares se torna relevante.

Una de estas potenciales patologías es la Ehrlichiosis reconocida como emergente y zoonotica por nuestra autoridad sanitaria.

El presente estudio se realizó con el objetivo de identificar la prevalencia de Anaplasma phagocytophilum en equinos Fina Sangre de Carrera (FSC) pertenecientes al Valparaíso Sporting Club, Viña del Mar, Chile.

Del lugar se tomaron muestras sanguíneas de 50 caballos FSC elegidos aleatoriamente durante los meses de Septiembre a Noviembre del 2011. Las muestras fueron evaluadas con un kit diagnóstico de Inmunofluorescencia indirecta (IFI) en el laboratorio CAMPVSVet, ubicado en Santiago de Chile.

De los 50 ejemplares analizados un 58\% (29) correspondieron a machos y un $42 \%$ (21) a hembras y las edades variaron de los 2 a 7 años. Del total de muestras se encontró un 8\% (4) de positividad a Anaplasma phagocytophilum en la prueba IFI.

Se establece con este estudio que la Ehrlichiosis Equina prevalente en la zona central de Chile es la Ehrlichiosis Granulocítica Equina.
\end{abstract}

